\title{
THE REGIONALLY PROXIMAL RELATION
}

\author{
JOSEPH AUSLANDER, DAVID B. ELLIS, AND ROBERT ELLIS
}

\begin{abstract}
Sufficient conditions for the regionally proximal relation $Q(X)$ of a minimal flow to be an equivalence relation are obtained in terms of the group $\mathscr{G}(X)$ of the flow and various groups which depend only on the acting group $T$.
\end{abstract}

\section{INTRODUCTION}

One of the first problems in topological dynamics was to characterize the equicontinuous structure relation $S(X)$ of a flow $(X, T)$; i.e. to find the smallest closed equivalence relation, $S(X)$ on $(X, T)$, such that $(X / S, T)$ is equicontinuous. A natural candidate for $S$ is the so-called regionally proximal relation $Q(X)$ (see below for the definition). Now $Q$ turns out to be closed, invariant, and reflexive, but not necessarily transitive. The problem was then to find conditions under which $Q$ was an equivalence relation. Starting with Veech [V], various authors, including MacMahon [M], Ellis-Keynes [EK], came up with various sufficient conditions for $Q$ to be an equivalence relation.

In this paper we give a sufficient condition in terms of the group of the flow $(X, T)$, which generalizes most of the ones previously adduced (see 1.12). These groups, introduced in [E1], have begun to play a fundamental role in topological dynamics in that many dynamical properties of flows may be characterized using them. Indeed 1.10, the principal result of this paper, may be viewed as saying that $Q(X)$ is an equivalence relation if $E \subset G^{\prime} \mathscr{G}(X)$ (see 1.12), a statement which refers only to the group $\mathscr{G}(X)$ of the flow $(X, T)$ and certain groups $G^{\prime}$ and $E$ which depend only on the acting group $T$.

The authors would like to thank Daniel Penazzi for many helpful discussions during the preparation of this paper.

We begin by recalling the definition of the regionally proximal relation $Q$.

1.1. Definition. Let $(X, T)$ be a flow. We define the regionally proximal relation $Q(X) \subset X \times X$ by

$$
\begin{array}{r}
Q(X)=\left\{(x, y) \mid \text { there exist } x_{i} \rightarrow x, y_{i} \rightarrow y, \text { and } t_{i} \in T\right. \\
\text { such that } \left.\lim x_{i} t_{i}=\lim y_{i} t_{i}\right\} .
\end{array}
$$

It is easily checked that $Q(X)$ is symmetric, closed, and $T$-invariant. Moreover $Q(X)$ is trivial if and only if the flow $(X, T)$ is equicontinuous.

Received by the editors August 8,1993; originally communicated to the Proceedings of the AMS by Barbara L. Keyfitz.

1991 Mathematics Subject Classification. Primary 54H20. 
1.2. Notation. Let $M$ be a fixed minimal right ideal in $\beta T$. We will write

$$
J=\left\{v \in M \mid v^{2}=v\right\} \text { and } G=M u,
$$

where $u \in J$ is fixed. Then $(M, u)$ is a universal pointed minimal $T$-flow; that is, given a minimal flow $(X, T)$ with base point $x_{0}=x_{0} u$, the map

$$
p \rightarrow x_{0} p: M \rightarrow X
$$

is a homomorphism of flows. We will write $Q$ for the regionally proximal relation $Q(M)$ and

$$
Q[p]=\{q \in M \mid(p, q) \in Q\} .
$$

Note that $G$ is a group which we equip with the $\tau$-topology. Recall that if $K \subset G$, then its closure in this topology is given by

$$
\operatorname{cls}_{\tau} K=(K \circ u) \cap G \text {. }
$$

Here

$$
K \circ u=\left\{p \in M \mid \text { there exist } p_{i} \in K \text { and } t_{i} \rightarrow u \text { with } p_{i} t_{i} \rightarrow p\right\} .
$$

A net $\left\{\alpha_{i}\right\} \subset G$ converges to $\alpha \in G$ in the $\tau$-topology if and only if there exists a net $\left\{t_{i}\right\} \subset T$ such that $t_{i} \rightarrow u$ and $\alpha_{i} t_{i} \rightarrow \alpha$ in the ordinary topology on $M$. We denote by $G^{\prime}$ the $\tau$-closed subgroup of $G$ defined by

$$
G^{\prime}=\bigcap\left\{\operatorname{cls}_{\tau} N \mid N \text { is a } \tau \text {-neighborhood of } u\right\} .
$$

It follows from the definitions that $\alpha \in G^{\prime}$ if and only if there exists a net in $G$ which converges to both $\alpha$ and $u$ in the $\tau$-topology.

We will need the following elementary property of $Q$ which we isolate as a lemma.

1.3. Lemma. Let $p \in M$ and $\left(q_{1}, q_{2}\right) \in Q$. Then $\left(p q_{1}, p q_{2}\right) \in Q$.

Proof. The map $q \rightarrow p q$ from $M \rightarrow M$ is a flow automorphism.

The next lemma deals with the relationship between $Q$ and $Q(X)$ for a flow $(X, T)$.

1.4. Lemma. Let $(X, T)$ be a minimal flow with base point $x_{0}=x_{0} u$. Let $(x, y) \in Q(X)$ with $x, y \in X w$, where $w \in J$. Suppose that $p \in M w$ with $x_{0} p=x$. Then there exists $q \in M w$ such that $x_{0} q=y$ and $(p, q) \in Q$.

Proof. It is well known (see [E2]) that there exist $\left(p_{0}, q_{0}\right) \in Q$ such that $x_{0} p_{0}=x_{0} p$ and $x_{0} q_{0}=y$. Since $x, y \in X w$, we may assume that $p_{0}, q_{0} \in$ $M w$. (Otherwise replace $p_{0}, q_{0}$ by $\left.p_{0} w, q_{0} w\right)$. Now $x_{0} p_{0} u=x_{0} p u$, so $\alpha=p u\left(p_{0} u\right)^{-1} \in M u$ satisfies

$$
x_{0} \alpha=x_{0} \quad \text { and } \quad \alpha p_{0}=\alpha p_{0} u w=p u w=p .
$$

It then follows from 1.3 that the pair $\left(\alpha p_{0}, \alpha q_{0}\right)=\left(p, \alpha q_{0}\right)$ satisfies the desired conditions.

1.5. Definition. We define a subset $H=H_{Q}$ of $G$ by

$$
H=Q[u] \cap G=\{\alpha \in G \mid(u, \alpha) \in Q\} .
$$

We gather a few properties of $H$. Although most of them follow readily from the definitions, in the interest of completeness we include their proofs. 
1.6. Lemma. (a) $G^{\prime} \subset H$,

(b) $H$ is $\tau$-closed,

(c) $H=H^{-1}$,

(d) $H \beta=\beta H$ for all $\beta \in G$,

(e) $H p=Q[p] \cap G v$ for all $p \in M v$.

Proof. (a) Let $\alpha \in G^{\prime}$. Then there exists a net $\alpha_{i}$ in $G$ which converges to $\alpha$ and to $u$ in the $\tau$-topology. Thus there exist nets in $T$

$$
t_{i} \rightarrow u \text { and } s_{i} \rightarrow u
$$

such that

$$
\alpha_{i} t_{i} \rightarrow \alpha, \quad \alpha_{i} s_{i} \rightarrow u
$$

But then $\alpha s_{i} \rightarrow \alpha u=\alpha$; moreover

$$
\alpha s_{i}\left(s_{i}^{-1} t_{i}\right) \rightarrow \alpha u=\alpha \text { and } \alpha_{i} s_{i}\left(s_{i}^{-1} t_{i}\right) \rightarrow \alpha .
$$

Hence $(u, \alpha) \in Q$ and $\alpha \in H$.

(b) Let $q \in H \circ p$. Then there exist $\alpha_{i} \in H$ and $t_{i} \in T$ such that

$$
t_{i} \rightarrow p, \quad \alpha_{i} t_{i} \rightarrow q .
$$

Now $\left(u, \alpha_{i}\right) \in Q$, so $\lim \left(u t_{i}, \alpha_{i} t_{i}\right)=(p, q) \in Q$. That is, $q \in Q[p]$ and hence $H \circ p \subset Q[p]$. It follows that

$$
\operatorname{cls}_{\tau} H=(H \circ u) \cap G \subset Q[u] \cap G=H .
$$

(c) follows immediately from the fact that $Q$ is symmetric.

(d) follows from 1.3 and the fact that $Q$ is $T$-invariant.

(e) Using the proof of part (b) we have

$$
H p \subset H \circ p \subset Q[p]
$$

for all $p \in M$. If $p \in M v$, then $H p=H p v \subset M v=G v$. On the other hand if $q \in Q[p] \cap G v$, then $(p, q) \in Q$ and hence $(p u, q u) \in Q$. It follows that

$$
h=q u(p u)^{-1} \in H \text {. }
$$

Thus $q=q u v=h p u v=h p v=h p \in H p$.

1.7. Definition. Let $p \in M$ and $v \in J$ with $p v=p$. Recall that the collection

$$
\{\bar{V} \mid V \subset T \text { with } p \in \bar{V}\}
$$

forms a neighborhood base for $p \in \beta T$. We define

$$
L(p)=\bigcap_{p \in \bar{V}} \operatorname{cls}_{\tau} \operatorname{int}_{\tau} \operatorname{cls}_{\tau}((M \cap \bar{V}) v) .
$$

We now prove a key technical lemma regarding $L(p)$.

\subsection{Lemma. Let $p \in M$. Then}

(a) $L(p) \neq \varnothing$,

(b) $G^{\prime} L(p)=L(p)$,

(c) $L(p) w \subset Q[p] \cap G w$ for all $w \in J$.

Proof. (a) Let $\varnothing \neq W \subset M$ be open. We consider the left action

$$
\begin{gathered}
G \times M \rightarrow M \\
(\alpha, q) \rightarrow \alpha q .
\end{gathered}
$$


A point $q \in M$ is almost periodic with respect to this action if and only if $q t$ is almost periodic for every $t \in T$. Thus the almost periodic points for this action are dense in $M$, and we can find $q \in W$ which is almost periodic. Now $q=q w$ for some $w \in J$ and

$$
\overline{G q}=\overline{G q w}=\overline{G w}
$$

is minimal with respect to the left action of $G$. Thus there exists a finite set $F \subset G w$ such that

$$
\overline{G w} \subset F(W \cap \overline{G w}) .
$$

But then

$$
G w \subset F(W \cap G w) \subset F \operatorname{cls}_{\tau}(W \cap G w) .
$$

Hence $\operatorname{int}_{\tau} \operatorname{cls}_{\tau}(W \cap G w) \neq \varnothing$. Since the map $\beta \rightarrow \beta v: G w \rightarrow G v$ is a $\tau$-homeomorphism for any idempotent $v \in J$, we have

$$
\varnothing \neq\left(\operatorname{int}_{\tau} \operatorname{cls}_{\tau}(W \cap G w)\right) v=\operatorname{int}_{\tau} \operatorname{cls}_{\tau}(W \cap G w) v \subset \text { int }_{\tau} \operatorname{cls}_{\tau} W v .
$$

The desired result now follows immediately from the finite intersection property.

(b) Let $v \in J$ with $p v=p$, and let $W \subset G v$ be a $\tau$-open subset. We include a proof that $G^{\prime} \operatorname{cls}_{\tau} W=\operatorname{cls}_{\tau} W$ although this result is found in [E2]. Let $\beta \in W$ and $\alpha \in G^{\prime}$. Then there exist a net $\alpha_{i} \in G v$ which converges to both $\alpha v$ and $v$ in the $\tau$-topology. Then $\alpha_{i} \beta$ converges to $\beta$ and hence we may assume that $\alpha_{i} \beta \in W$ for all $i$. But $\alpha_{i} \beta$ also converges to $\alpha \beta$, so $\alpha \beta \in \operatorname{cls}_{\tau} W$. We have shown that

$$
G^{\prime} W \subset \operatorname{cls}_{\tau} W
$$

from which it follows immediately that $G^{\prime} \operatorname{cls}_{\tau} W=\operatorname{cls}_{\tau} W$. Applying this to int $_{\tau} \operatorname{cls}_{\tau}((M \cap \bar{V}) v)$ yields the desired result.

(c) First note that for any neighborhood $N$ of $p$ in $M$

$$
\begin{aligned}
L(p) w & \subset \operatorname{cls}_{\tau} \operatorname{int}_{\tau} \operatorname{cls}_{\tau}(N w) \subset \operatorname{cls}_{\tau}(N w) \\
& =(N w \circ w) \cap G w \subset((N \circ w) \circ w) \cap G w=(N \circ w) \cap G w .
\end{aligned}
$$

Let $r \in L(p) w$, so $r \in N \circ w$ for any neighborhood in $N$ of $p$. Thus there exist nets $p_{i} \rightarrow p, t_{i} \rightarrow w$ with $p_{i} t_{i} \rightarrow r$. On the other hand $r t_{i} \rightarrow r w=r$, so $(p, r) \in Q$.

1.9. Proposition. Let $A$ be a $\tau$-closed subgroup of $G$ such that $A H$ is a group. Then $A H p=A H L(p)$ for all $p \in M$.

Proof. $A H L(p)$ is the union of a collection of cosets of $A H$, so it suffices to show that $L(p) \subset A H p$. Let $v \in J$ with $p v=p$. Then by 1.8 and 1.6 $L(p)=L(p) v \subset Q[p] \cap G v=H p$.

Let $(X, T)$ be a minimal flow with basepoint $x_{0}$ satisfying $x_{0} u=x_{0}$. The so-called group of the flow

$$
\mathscr{G}(X, T)=\left\{\alpha \in G \mid x_{0} \alpha=x_{0}\right\} .
$$

Our main theorem gives a condition on $\mathscr{G}(X, T)$ which guarantees that $Q(X)$ is an equivalence relation. 
1.10. Theorem. Let $(X, T)$ be a minimal flow with group $A=\mathscr{G}(X, T)$, and assume that $H \subset A G^{\prime}$. Then $Q$ is an equivalence relation on $X$.

Proof. Let $(x, y),(y, z) \in Q(X)$. By $1.6 G^{\prime} \subset H$, so the assumption that $H \subset A G^{\prime}$ is equivalent to the statement $A H=A G^{\prime}$. In particular $A H$ is a group. We first show, using only the assumption that $A H$ is a group, that $z=x h v$ for some $h \in H$ and $v \in J$. We then complete the proof (using the fact that $\left.A H=A G^{\prime}\right)$ by showing that $(x, z) \in Q(X)$.

The flow $(X, T)$ is minimal, so there exist $w, v \in J$ such that $x w=x$ and $z v=z$. Thus $(x w, y w)=(x, y w)$ and $(y w, z w)$ are both in $Q(X)$. Applying 1.4 there exist $\beta_{1}, \beta_{2}, \beta_{3} \in G w$ with

$$
\left(\beta_{1}, \beta_{2}\right),\left(\beta_{2}, \beta_{3}\right) \in Q \text { and } x_{0} \beta_{1}=x, x_{0} \beta_{2}=y w, x_{0} \beta_{3}=z w .
$$

We thus have

$$
\beta_{2} \beta_{1}^{-1}, \beta_{3} \beta_{2}^{-1} \in Q[w] \cap G w=H w .
$$

The fact that $A H$ and hence $A H w$ are groups now implies that $\beta_{3} \beta_{1}^{-1} \in A H w$ and hence $\beta_{3} \in A H \beta_{1}=A \beta_{1} H w$. It follows that there exists $h \in H$ with $x_{0} \beta_{3}=x_{0} \beta_{1} h w$. Then

$$
z=z v=z w v=x_{0} \beta_{3} v=x_{0} \beta_{1} h w v=x h v .
$$

To complete the proof we observe that

$$
\begin{aligned}
v & =u v \in\left(\beta_{1}^{-1} A H \beta_{1}\right) v=\left(\beta_{1}^{-1} A \beta_{1}\right) H v \\
& =\left(\beta_{1}^{-1} A \beta_{1}\right) H(h v)=\left(\beta_{1}^{-1} A \beta_{1}\right) H L(h v) \\
& =\left(\beta_{1}^{-1} A H \beta_{1}\right) L(h v)=\left(\beta_{1}^{-1} A G^{\prime} \beta_{1}\right) L(h v) \\
& =\left(\beta_{1}^{-1} A \beta_{1}\right) G^{\prime} L(h v)=\left(\beta_{1}^{-1} A \beta_{1}\right) L(h v) .
\end{aligned}
$$

Thus

$$
u=v u \in\left(\beta_{1}^{-1} A \beta_{1}\right)(L(h v) u) \subset\left(\beta_{1}^{-1} A \beta_{1}\right) Q[h v]
$$

and hence

$$
\left(\beta_{1}^{-1} \beta \beta_{1}, h v\right) \in Q
$$

for some $\beta \in A$. We then have $\left(\beta \beta_{1}, \beta_{1} h v\right) \in Q$ by 1.3 , and

$$
(x, z)=\left(x_{0} \beta_{1}, x h v\right)=\left(x_{0} \beta \beta_{1}, x_{0} \beta_{1} h v\right) \in Q(X)
$$

as desired.

We shall see that the converse of 1.10 is false, but first we deduce some interesting consequences.

1.11. Proposition. Let $A$ be a $\tau$-closed subgroup of $G$. Then the $\tau$-closed subgroup generated by $A H$ is $A E$ where $E$ is the group of the universal equicontinuous minimal flow.

Proof. Let $B$ be the $\tau$-closed subgroup of $G$ generated by $A H$. Since $Q$ is contained in the equicontinuous structure relation on $M, H \subset E$ and hence $B \subset A E$. There exists a minimal flow $(X, T)$ such that $\mathscr{G}(X, T)=B$ (see [E2]). Now $H \subset B=B G^{\prime}$, so $Q(X)$ is an equivalence relation by 1.10 . Thus $Q(X)$ is the equicontinuous structure relation on $X$ (see [A]). In other words the maximal equicontinuous factor of $X$ is given by

$$
X_{\mathrm{eq}}=X / Q(X) \text {. }
$$


The group $\mathscr{G}\left(X_{\mathrm{eq}}, T\right)=B E$. On the other hand it follows from (*) that

$$
\begin{aligned}
\mathscr{G}\left(X_{\mathrm{eq}}, T\right) & =\left\{\alpha \in G \mid\left(x_{0}, x_{0} \alpha\right) \in Q(X)\right\} \\
& =\left\{\alpha \in G \mid x_{0} \alpha=x_{0} h \text { for some } h \in H\right\} \\
& =B H=B .
\end{aligned}
$$

Hence $B E=B$, so $A E \subset B$.

In the following proposition, $(X, T)$ is a minimal flow with basepoint $x_{0}=$ $x_{0} u$ and $A=\mathscr{G}(X, T) . P(X)$ denotes the proximal relation on $X$, and $S(X)$ denotes the equicontinuous structure relation on $X$.

1.12. Proposition. The following are equivalent:

(1) $A H$ is a group,

(2) $A H=A E$,

(3) $S(X)=Q(X) P(X)$,

(4) $Q(X) P(X)$ is a closed equivalence relation.

Proof. (1) $\Rightarrow(2)$ is an immediate consequence of Proposition 1.11, and (2) $\Rightarrow$ (1) is obvious.

(3) $\Rightarrow(2)$ It is sufficient to show that $E \subset A H$. Let $\varepsilon \in E$. Then $\left(x_{0}, x_{0} \varepsilon\right) \in$ $S(X)$, so $\left(x_{0}, y\right) \in Q(X)$ and $\left(y, x_{0} \varepsilon\right) \in P(X)$ for some $y \in X$. Hence there exists a minimal right ideal $I \subset \beta T$ such that

$$
y r=x_{0} \varepsilon r \quad \text { for all } r \in I .
$$

Let $w \in I$ be an idempotent such that $u w=u$ and $w u=w$. (For a proof that such an idempotent exists see [E2].) Then

$$
\begin{aligned}
\left(x_{0}, x_{0} \varepsilon\right) & =\left(x_{0} u, x_{0} \varepsilon u\right)=\left(x_{0} u w, x_{0} \varepsilon u w\right) \\
& =\left(x_{0} w, x_{0} \varepsilon w\right)=\left(x_{0} w, y w\right) \in Q(X),
\end{aligned}
$$

and it follows that $\varepsilon \in A H$.

(2) $\Rightarrow$ (3) Clearly $Q(X) P(X) \subset S(X)$. Suppose that $\left(x_{0}, y_{0}\right) \in S(X)$ and that $v \in \beta T$ is a minimal idempotent with $y_{0} v=y_{0}$. Then

$$
y_{0} u=x_{0} \varepsilon \text { for some } \varepsilon \in E \text {; }
$$

and since we are assuming that $A H=A E$, there exists $h \in H$ with $x_{0} \varepsilon=x_{0} h$. Now

$$
\left(x_{0}, x_{0} h\right) \in Q(X) \text { and }\left(x_{0} h, x_{0} h v\right) \in P(X),
$$

so $\left(x_{0}, y_{0}\right)=\left(x_{0}, y_{0} u v\right)=\left(x_{0}, x_{0} h v\right) \in Q(X) P(X)$. In particular note that if $y_{0} u=y_{0}$ we have shown that $\left(x_{0}, y_{0}\right) \in Q(X)$.

Now suppose that $(x, y) \in S(X)$ with $x w=x$ where $w \in \beta T$ is a minimal idempotent. Let $\alpha \in G$ such that $x \alpha=x_{0}$. Then as was noted above we have

$$
\left(x_{0}, y \alpha\right)=(x \alpha, y \alpha) \in Q(X) \text {. }
$$

Thus

$$
(x, y w)=\left(x_{0} \alpha^{-1} w,(y \alpha) \alpha^{-1} w\right) \in Q(X) .
$$

Since $(y w, y) \in P(X)$, it follows that $(x, y) \in Q(X) P(X)$.

(4) $\Rightarrow$ (3) The relation $Q(X) P(X)$ is $T$-invariant, contains $Q(X)$, is contained in $S(X)$, and so must be $S(X)$.

$(3) \Rightarrow(4)$ is obvious. 
1.13. Remarks. (1) In view of $1.11,1.10$ is equivalent to: $E \subset A G^{\prime}$ implies $Q(X)$ is an equivalence relation. Since this statement involves only the group $A$ of the flow, it is natural to ask whether $Q(X)$ being an equivalence relation is a "group condition"; i.e., given that $\mathscr{G}(X, T)=\mathscr{G}(Y, T)$ and that $Q(X)$ is an equivalence relation does it follow that $Q(Y)$ is one also?

(2) In [E3] it is shown that when the almost periodic points are dense in $X \times X, E \subset A G^{\prime}$. Thus 1.10 generalizes the result that $Q(X)$ is an equivalence relation when the almost periodic points are dense in $X \times X$. In particular, if $(X, T)$ is point distal, then the almost periodic points are dense in $X \times X$ and it follows from 1.10 that $Q$ is an equivalence relation for any proximal extension of $(X, T)$.

(3) The proof of the crucial lemma 1.8 raises the question: Is every $p \in M$ an almost periodic point of the flow $(G, M)$, and if not why not?

The following lemma follows from a combination of Proposition 14.14 of [E2] and Proposition 3.10 of [EGS]. In the interest of completeness we include an outline of a (different) proof.

1.14. Lemma. Let $D$ be the group of the universal distal minimal flow. Then $G^{\prime} D=E$.

Proof. First note that $G^{\prime} \subset H \subset E$ and $D \subset E$ (because the universal equicontinuous flow is a factor of the universal distal flow). Thus $G^{\prime} D \subset E$. Let $B$ be any $\tau$-closed normal subgroup of $G$ which contains $G^{\prime} D$. Then the map

$$
t \rightarrow u t u: T \rightarrow G / B
$$

is a homomorphism. Indeed $G / B$ is a compact topological group, so $(G / B, T)$ is an equicontinuous flow. Applying this to $B=G^{\prime} D$ and $B=E$ we obtain $G / G^{\prime} D \rightarrow G / E$, a homomorphism of equicontinuous flows. But $G / E$ is the maximal equicontinuous flow, so $G^{\prime} D=E$.

1.15. Corollary. Let $(X, T)$ be a minimal flow for which the proximal relation is closed. Then $Q(X)$ is an equivalence relation.

Proof. Since the proximal relation is closed, it must be an equivalence relation (see [A]). Dividing out by this relation we see that $(X, T)$ is a proximal extension of a distal flow. Hence $D \subset \mathscr{G}(X, T)=A$, which implies (by 1.14) that $H \subset E=D G^{\prime} \subset A G^{\prime}$. It then follows from 1.10 that $Q(X)$ is an equivalence relation.

We now give an example which shows that the converse of Theorem 1.10 is false.

1.16. Example. Let $X=S^{n-1}$ and $T=S L(n, \mathbf{R})$ with $n \geq 2$. We consider the action

$$
\begin{aligned}
& X \times T \rightarrow X, \\
& (x, l) \rightarrow \frac{x l}{\|x l\|} .
\end{aligned}
$$

Then $Q$ is an equivalence relation on $X$; in fact $(x, y) \in Q(X)$ for all $x, y \in$ $X$. Moreover van der Waerden (see [W]) shows that the constants are the only almost periodic functions on $T$ when the latter is provided with the discrete topology; hence $E=G$ in this case. On the other hand the identification of antipodal points gives a $Z_{2}$ group extension:

$$
X=S^{n-1} \rightarrow \mathbf{R P}^{n} \text {. }
$$


But $\left(\mathbf{R P}^{n}, T\right)$ is proximal, so $\mathscr{G}\left(\mathbf{R P}^{n}, T\right)=G$. Therefore $G / \mathscr{G}(X, T) \cong \mathbf{Z}_{2}$ and $A=\mathscr{G}(X, T)$ is of index 2 in $G$. It follows that $A$ is $\tau$-clopen and $G^{\prime} \subset A \neq G$. Now the fact that $Q(X)=X \times X$ implies that $A H=G$. Hence $H \not \subset A=A G^{\prime}$.

\section{REFERENCES}

[A] J. Auslander, Minimal flows and their extensions, North-Holland, Amsterdam, 1988.

[E1] R. Ellis, Group-like extensions of minimal sets, Trans. Amer. Math. Soc. 127 (1967), 125-135.

[E2] L Lectures on topological dynamics, Benjamin, New York, 1969.

[E3] — The Veech structure theorem, Trans. Amer. Math. Soc. 186 (1973), 203-218.

[EGS] R. Ellis, S. Glasner, and L. Shapiro, Proximal-isometric (PI) flows, Adv. in Math. 17 (1975), 213-260.

[EK] R. Ellis and H. Keynes, $A$ characterization of the equicontinuous structure relation, Trans. Amer. Math. Soc. 161 (1971), 171-181.

[M] D. C. McMahon, Relativized weak disjointness and relatively invariant measures, Trans. Amer. Math. Soc. 236 (1978), 225-237.

[V] W. A. Veech, The equicontinuous structure relation for minimal abelian transformation groups, Amer. J. Math. 90 (1968), 723-732.

[W] B. L. van der Waerden, Stetigkeitssatz der halbeinfachen Lieschen Gruppen, Math. Z. 161 (1933), 780-786.

Department of Mathematics, University of Maryland, College Park, Maryland 20742

Department of Mathematics, Beloit College, Beloit, Wisconsin 53511

Department of Mathematics, University of Minnesota, Minneapolis, Minnesota 55455 